\title{
ISOMETRIC FLOWS ON HILBERT SPACE
}

\author{
BY P. MASANI ${ }^{1}$
}

Communicated by Paul R. Halmos, July 25, 1962

1. Introduction. It is known that if $V$ is an isometry on a (complex) Hilbert space $X$ onto a subspace $R$ of $X$, then

$$
X=\sum_{k=0}^{\infty} V^{k}\left(R^{\perp}\right)+\bigcap_{k=0}^{\infty} V^{k}(x),
$$

where the two subspaces on the right-hand side are orthogonal, and $R^{\perp}$ is "wandering for $V$, i.e. $V^{j}\left(R^{\perp}\right) \perp V^{k}\left(R^{\perp}\right), j \neq k .{ }^{2}$ The identity (1.1) closely resembles the Wold decomposition of the "present and past subspace" of a weakly stationary stochastic process into its "innovation subspaces" and the "remote past" cf. [10, 6.10]. Interpreting $k$ as the time, we shall therefore speak of (1.1) as the Wold decomposition of $x$ due to $V$ or (equivalently) due to the discrete semi-group ( $V^{k}$, $k \geqq 0)$, and refer to $V^{k}\left(R^{\perp}\right), k \geqq 0$, as the innovation subspaces of $X$, and to $\bigcap_{k=0}^{\infty} V^{k}(X)$ as the remote subspace of $x$ engendered by the semi-group.

In this note our purpose is to obtain the analogous decomposition of $x$ due to a strongly continuous semi-group $\left(S_{t}, t \geqq 0\right)$ of isometries on $x$ into $x$ ( 6.5 below). We shall derive this by applying (1.1) to the Cayley transform $V$ of $H$, where $i H$ is the infinitesimal generator of the semi-group, and then replacing the direct sum $\sum_{k=0}^{\infty} V^{k}\left(R^{\perp}\right)$ of innovation subspaces, occurring in (1.1), by a direct integral of "differential innovation subspaces."

2. The associated discrete semi-group. Let $\left(S_{t}, t \geqq 0\right)$ be a strongly continuous semi-group of isometries on $X$ into $X$, and let $i H$ be its infinitesimal generator. Then

$$
S_{t}^{\prime}=S_{t} i H=i H S_{t}, \quad \text { on } D, \quad t \geqq 0,
$$

where $D$, the domain of $H$, is a linear manifold everywhere dense in $x$. From the work of J. L. B. Cooper [1], (cf. also [5]) ${ }^{3}$ we know that

(a) $H$ is maximal symmetric with deficiency index $(0, \alpha)$,

1 This work was supported by the Office of Naval Research.

2 This result, implicit in the work of von Neumann and Murray on rings of operators, is proved and put to significant use in a recent paper by Halmos [4] (cf. also [6]).

${ }^{3}$ Our approach differs from Cooper's in that we make systematic use of the operator $T_{a, b}$ defined in (4.1), and of the deficiency subspace $R^{\perp}$ of $H$. 
(b) $H+i I$ is one-one on $D$ onto $x$,

(c) $(H+i I)^{-1}=\frac{1}{i} \int_{0}^{\infty} e^{-t} S_{t} d t$ is one-one and bounded on $x$ onto

$D$ and $\left|(H+i I)^{-1}\right| \leqq 1,4$

(d) $H-i I$ is one-one on D onto a (closed) subspace $R .^{5}$

Now let $V$ be the Cayley transform of $H$ :

$$
V=c(H)=(H-i I)(H+i I)^{-1}, \quad \text { on } \boldsymbol{X} .
$$

It follows from the work of von Neumann, cf. [9, Chapter IX], that

(a) $V$ is an isometry on $x$ onto $R$,

$$
\begin{aligned}
& \text { (b) } I-V=2 i(H+i I)^{-1}=2 \int_{0}^{\infty} e^{-t} S_{t} d t \quad \text { on } x \text {, } \\
& \text { (c) } H=i(I+V)(I-V)^{-1} \text { on } D, \\
& \text { (d) } S_{t} V^{k}=V^{k} S_{t} \quad \text { on } x, \quad t \geqq 0, k \geqq 0 .
\end{aligned}
$$

We shall call ( $V^{k}, k \geqq 0$ ) the discrete semi-group of isometries associated with the given semi-group $\left(S_{t}, t \geqq 0\right)$. In the rest of $\S 2$ we shall formulate the basic relationship between the $S_{t}$ and the $V^{k}$.

The $S_{t}$ are expressible in terms of $H$ by the exponential formula, cf. [5],

$$
S_{t}=\lim _{n \rightarrow \infty} \exp \left(t i H J_{n}\right), \quad \text { strongly on } X \text {, }
$$

$$
\text { where } J_{n}=\left(I-\frac{1}{n} i H\right)^{-1} \text {. }
$$

Since $J_{n}$ is a bounded operator, so therefore is $i H J_{n}=n\left(J_{n}-I\right)$. Hence $\exp \left(t i H J_{n}\right)$ has a power series expansion, from which we get the following expression for $S_{t}$ in terms of $V^{k}$ :

$$
\begin{aligned}
& S_{t}=e^{-t} I+\lim _{n \rightarrow \infty} \sum_{k=1}^{\infty}\left\{\frac{1}{k !}\left(\frac{-n t}{n+1}\right)^{k} \sum_{j=1}^{k}\left(\begin{array}{c}
k \\
j
\end{array}\right) K_{n}^{j}\right\}, \quad t \geqq 0, \\
& K_{n}=\frac{2 n}{n+1}\left\{I-\frac{n-1}{n+1} V\right\}^{-1} V, \quad \text { and } \quad \text { so } \quad K_{n}(x) \subseteq R, \quad n \geqq 1 \text {. }
\end{aligned}
$$

Reciprocally, we find from (2.3)(b) the following expression for $V^{n}$ in terms of the $S_{t}$ :

4 The absolute value sign refers to the usual Banach norm of the operator.

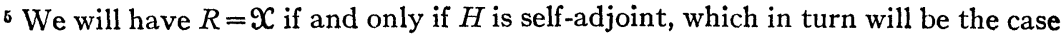
if and only if the isometries $S_{t}$ are actually unitary. For us this is the uninteresting case in which the Wold decomposition reduces to the triviality $X=X$. 


$$
\begin{aligned}
V^{n} & =I+2 \int_{0}^{\infty} L_{n}^{\prime}(2 t) e^{-t} S_{t} d t, \\
L_{n}(t) & =\sum_{k=0}^{n} \frac{(-1)^{k}}{k !}\left(\begin{array}{l}
n \\
k
\end{array}\right) t^{k}, \quad \text { (nth Laguerre polynomial, [8]). }
\end{aligned}
$$

From (2.5), (2.6) we get the following useful identity between the subspaces generated by the sets $S_{t}(X), t \geqq 0$, and $V^{k}(X), k \geqq 0$ :

$$
\Im\left\{S_{t}(X)\right\}_{t \geq 0}=\subseteq\left\{V^{k}(X)\right\}_{k \geq 0}, \quad X \subseteq x .
$$

From (2.5) we also see that

$$
S_{t}(x)=e^{-t} x+y_{t}, \quad y_{t} \in R, t \geqq 0, x \in X,
$$

and hence

$$
\begin{aligned}
\left(S_{t}(x), y\right) & =e^{-t}(x, y), \quad x \in x, y \in R^{\perp}, t \geqq 0, \\
\left(S_{s}(x), S_{t}(y)\right) & =e^{-|s-t|}(x, y), \quad x, y \in R^{\perp}, s, t \geqq 0,
\end{aligned}
$$

where $($,$) denotes the inner product in x$.

3. The remote subspace. Let us write

$$
\begin{array}{ll}
x_{t}=S_{t}(x), & x_{k}^{\prime}=V^{k}(x), \quad t, k \geqq 0, \\
x_{\infty}=\bigcap_{t \geqq 0} x_{t}, & x_{\infty}^{\prime}=\bigcap_{k \geq 0} x_{k}^{\prime} .
\end{array}
$$

We assert the following crucial theorem:

3.2. TheOREM. $x_{\infty}=X_{\infty}^{\prime}$. The restrictions of the isometries $S_{t}, V^{n}$, for $t, n \geqq 0$, to the subspace $x_{\infty}$ are unitary.

To prove this we first show, quite easily, that the restrictions of $S_{t}$ and $V^{k}$ to the remote subspaces $X_{\infty}, \mathscr{X}_{\infty}^{\prime}$, respectively, are unitary. We then establish the deeper result $x_{\infty}=X_{\infty}^{\prime}$. The inclusion $x_{\infty} \subseteq X_{\infty}^{\prime}$ follows without much difficulty from (2.8) and (2.3). The reverse inclusion requires the following lemma, which rests on the fact that $D$ is the range of $I-V$, and on the limiting behavior of $L_{n}(t)$, as $n \rightarrow \infty$, cf. [8, pp. 333-334]:

Lemma. Let $\mathfrak{D}_{\infty}^{\prime}=\bigcap_{k \geqq 0} V^{k}(D)$, where $D$ is the domain of the infinitesimal generator $i H$. Then

(a) $D_{\infty}^{\prime}$ is a linear manifold everywhere dense in $\mathfrak{X}_{\infty}^{\prime}$,

(b) $\mathfrak{D}_{\infty}^{\prime} \subseteq X_{\infty}$ (and so $\mathfrak{X}_{\infty}^{\prime}=$ clos. $\mathscr{D}_{\infty}^{\prime} \subseteq X_{\infty}$ ).

Let us take the Wold decomposition (1.1) of $X$ due to $V=c(H)$, the Cayley transform of $H$. As just shown $\bigcap_{k=0}^{\infty} V^{k}(X)=x_{\infty}$. Also, on taking $X=R^{\perp}$ in (2.7) we find that $\sum_{k=0}^{\infty} V^{k}\left(R^{\perp}\right)=\mathfrak{S}\left\{V^{k}\left(R^{\perp}\right)\right\}_{k \geq 0}$ $=\mathfrak{S}\left\{S_{t}\left(R^{\perp}\right)\right\}_{t \geq 0}$. Thus (1.1) reduces to 


$$
x=\mathfrak{S}\left\{S_{t}\left(R^{\perp}\right)\right\}_{t \geq 0}+x_{\infty}, \quad \mathfrak{S}\left\{S_{t}\left(R^{\perp}\right)\right\}_{t \geq 0} \perp x_{\infty} .
$$

On applying $S_{a}$ we get

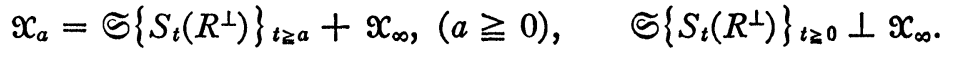

We shall refer to (3.3) as the pre-Wold decomposition of $x_{a}$ due to the semi-group $\left(S_{t}, t \geqq 0\right)$. Our task is to express the first subspace on the right-hand side as a direct integral of differential subspaces.

4. Differential innovation subspaces. We first introduce an operator-valued interval-measure. The measure $T_{a b}$ of the interval $[a, b], 0 \leqq a \leqq b$, is defined by

$$
T_{a b}=T_{b}-T_{a}, \text { where } T_{t}=\frac{1}{\sqrt{ } 2}\left\{S_{t}-I-\int_{0}^{t} S_{s} d s\right\}, \quad t \geqq 0 .
$$

We see at once that $T_{a b}, T_{t}$ are bounded linear operators on $X$ into $X$, that $T_{t}=T_{0 t}, t \geqq 0$, and that

(a) $T_{a b}+T_{b c}=T_{a c}, \quad 0 \leqq a \leqq b \leqq c$,

$$
\begin{aligned}
& \text { (b) } T_{a b}=\frac{1}{\sqrt{ } 2}\left\{S_{b}-S_{a}-\int_{a}^{b} S_{s} d s\right\}, \quad 0 \leqq a \leqq b \\
& \text { (c) } S_{t} T_{a b}=T_{a+t, b+t}, \quad 0 \leqq a \leqq b, 0 \leqq t
\end{aligned}
$$

By inverting the relations (4.1) we get the following expression for $S_{t}$ in terms of the $T_{\sigma \tau}$ :

$$
S_{t}=-\sqrt{ } 2 \int_{t}^{\infty} e^{t-s} T_{t s} d s=\sqrt{ } 2\left\{T_{t}-\int_{t}^{\infty} e^{t-s} T_{s} d s\right\} .
$$

We consider next the subspace-valued interval measure:

$$
\Re_{a b}=T_{a b}\left(R^{\perp}\right), \quad 0 \leqq a \leqq b .
$$

This has the following convenient properties, which are easy to check:

(a) $S_{t}\left(\Re_{a b}\right)=\Re_{a+t, b+t}, 0 \leqq a \leqq b, 0 \leqq t$;

(b) $\Re_{a b} \perp \Re_{c d}, 0 \leqq a<b \leqq c<d$;

(c) $\frac{1}{\sqrt{ }(b-a)} T_{a b}$ is an isometry on $R^{\perp}$ onto $\Re_{a b}, a<b$;

i.e. $\left(T_{a b} x, T_{a b} y\right)=(b-a)(x, y), x, y \in R^{\perp} ;$

(d) $\left(T_{J}(x), T_{K}(y)\right)=\left(T_{J \cap_{K}}(x), T_{J \cap_{K}}(y)\right)=|J \cap K|(x, y)$, where $x, y \in R^{\perp}, J, K$ are intervals and || is the length. 
From (4.5)(c) we see at once that

(4.6) $\mathfrak{N}_{a b}$ is a (closed) subspace of $X$, and $\operatorname{dim} . \Re_{a b}=\operatorname{dim} . R^{\perp}, \quad 0 \leqq a<b$.

But it should be noted that our subspace-valued measure $\Re_{a b}$ is only subadditive, i.e. $\mathscr{T}_{a c} \subset \mathscr{T}_{a b}+\mathfrak{N}_{b c}, 0 \leqq a<b<c$; for, we find that

$$
\mathfrak{K}_{a c}^{\perp} \cap\left(\mathscr{T}_{a b}+\mathfrak{T}_{b c}\right)=\left(\frac{1}{b-a} T_{a b}-\frac{1}{c-b} T_{b c}\right)\left(R^{\perp}\right),
$$

and the last is not $\{0\}$ even when $\operatorname{dim} . R^{\perp}=1$.

A simple but important consequence of $(4.2)(\mathrm{b})$ and (4.3) is the identity

$$
\Im\left\{S_{t}\left(R^{\perp}\right)\right\}_{t \geqq a}=\Im\left(\mathscr{T}_{s t}\right)_{a \leqq s<t<\infty}=\Im\left\{T_{s t}\left(R^{\perp}\right)\right\}_{a \leqq s<t<\infty} .
$$

This identity enables us to restate the pre-Wold decomposition (3.3) in the form

$$
\mathscr{X}_{a}=\Im\left\{T_{s t}\left(R^{\perp}\right)\right\} a_{\geqq s<t<\infty}+\mathscr{X}_{\infty}, \quad(a \geqq 0), T_{s t}\left(R^{\perp}\right) \perp X_{\infty} .
$$

On comparing this with the corresponding decomposition in the discrete case (cf. (1.1), (3.1)), viz.

$$
\mathfrak{X}_{n}=\mathfrak{S}\left\{V^{k}\left(R^{\perp}\right)\right\}_{k \geqq n}+\mathfrak{X}_{\infty}^{\prime},(n \geqq 0), \quad V^{k}\left(R^{\perp}\right) \perp \mathfrak{X}_{\infty}^{\prime},
$$

we see that the subspaces $T_{s t}\left(R^{\perp}\right)$ have taken the place of the "innovation subspaces" $V^{k}\left(R^{\perp}\right)$. This fact along with the properties (4.5)(b), (4.6) justifies our calling $T_{s t}\left(R^{\perp}\right), 0 \leqq s<t$, the differential innovation subspaces of $X$ engendered by the semi-group $\left(S_{t}, t \geqq 0\right)$.

Now in the discrete case we have the direct sum representation:

$$
\Im\left\{V^{k}\left(R^{\perp}\right)\right\}_{k \geq 0}=\sum_{k=0}^{\infty} V^{k}\left(R^{\perp}\right),
$$

where, by definition,

$$
\sum_{k=0}^{\infty} V^{k}\left(R^{\perp}\right)=\left\{\xi: \xi=\sum_{k=0}^{\infty} V^{k}\left(x_{k}\right), x_{k} \in R^{\perp} \& \sum_{k=0}^{\infty}\left|x_{k}\right|^{2}<\infty\right\} .
$$

This suggests that in the continuous case we should have an analogous direct integral representation:

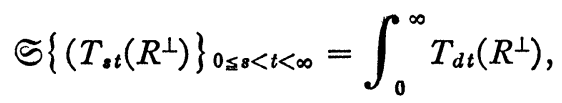

where

$$
\int_{0}^{\infty} T_{d t}\left(R^{\perp}\right)=\left\{\xi: \xi=\int_{0}^{\infty} T_{d t}\left(x_{t}\right), x_{t} \in R^{\perp} \& \int_{0}^{\infty}\left|x_{t}\right|^{2} d t<\infty\right\} .
$$


This heuristic reasoning can be put on a sound footing by defining precisely the vector-valued integral $\int_{0}^{\infty} T_{d t}\left(x_{t}\right)$ occurring in the last equation. This is done in $\$ \S 5,6$ below.

5. Generalized vector-valued integrals. Let $L_{2}\left([a, b], R^{\downarrow}\right)$ be the Hilbert space of all strongly (Lebesgue) measurable functions $x$ on $[a, b]$ with values $x_{t} \in R^{\perp}$ such that $\int_{a}^{b}\left|x_{t}\right|^{2} d t<\infty .{ }^{6}$ Our task is to define $\int_{a}^{b} T_{d t}\left(x_{t}\right)$ so that it will behave like a vector sum $\sum_{k=m}^{n} V^{k}\left(x_{k}\right)$, where $x_{k} \in R^{\perp}$. This suggests that we define it so as to ensure the following properties: for all functions $x, y, x^{(n)} \in L_{2}\left([a, b], R^{\perp}\right)$,
(a) $\left(\int_{a}^{b} T_{d t}\left(x_{t}\right), \int_{a}^{b} T_{d t}\left(y_{t}\right)\right)=\int_{a}^{b}\left(x_{t}, y_{t}\right) d t$,
(b) $\left|\int_{a}^{b} T_{d t}\left(x_{t}\right)\right|^{2}=\int_{a}^{b}\left|x_{t}\right|^{2} d t$,
(c) $\int_{a}^{b} T_{d t}\left(c x_{t}+d y_{t}\right)=c \int_{a}^{b} T_{d t}\left(x_{t}\right)+d \int_{a}^{b} T_{d t}\left(y_{t}\right)$,
(d) $\int_{a}^{b} T_{d t}\left(x_{t}^{(n)}\right) \rightarrow \int_{a}^{b} T_{d t}\left(x_{t}\right)$, when $x^{(n)} \rightarrow x$ in the $L_{2}$-topology.

The requisite definition consists of two parts, one for step-functions $x$ and the other for arbitrary $x$ in $L_{2}\left([a, b], R^{\perp}\right)$ :

5.2(a). Definition. For the step-function $x=\sum_{k=1}^{n} \alpha_{k} \chi_{J_{k}}$ on $[a, b]$, where $\alpha_{k} \in R^{\perp}$ and $\chi_{J_{k}}$ is the indicator-function of the bounded interval $J_{k}$ we define $\int_{a}^{b} T_{d t}\left(x_{t}\right)=\sum_{k=1}^{n} T_{J_{k}}\left(\alpha_{k}\right)$.

It follows from (4.5) that this definition is unequivocal and that the laws (5.1)(a)-(c) hold when $x$ and $y$ are step-functions. Moreover, for any Cauchy-sequence of step-functions $x^{(n)}$ in $L_{2}\left([a, b], R^{\perp}\right)$ we have

$$
\left|\int_{a}^{b} T_{d t}\left(x_{t}^{(m)}\right)-\int_{a}^{b} T_{d t}\left(x_{t}^{(n)}\right)\right|^{2}=\int_{a}^{b}\left|x_{t}^{(m)}-x_{t}^{(n)}\right|^{2} d t \rightarrow 0,
$$

as $m, n \rightarrow \infty$. This relation and the well-known fact that the stepfunctions are everywhere dense in $L_{2}\left([a, b], R^{\perp}\right)$ suggest the following extension of our definition:

5.2(b). Definition. For any $x \in L_{2}\left([a, b], R^{\perp}\right)$, we define $\int_{a}^{b} T_{d t}\left(x_{t}\right)$ $=\lim _{n \rightarrow \infty} \int_{a}^{b} T_{d t}\left(x_{t}^{(n)}\right)$, where $\left(x^{(n)}, n \geq 1\right)$ is any sequence of step-functions tending to $x$ in the $L_{2}$-topology.

It is easy to check that our definition is again unequivocal, and

${ }^{6}$ Cf. [3, Chapter III, §6]. According to their Theorem $6, L_{2}\left([a, b], R^{\perp}\right)$ is a Banach space. With the inner product $(x, y)=\int_{a}^{b}\left(x_{t}, y_{t}\right) d t$, it is obviously a Hilbert space. 
that the laws (5.1) hold without restriction. Moreover, as an intervalfunction the integral is seen to have the following properties:

$$
\begin{aligned}
& \text { (a) } \int_{a}^{b} T_{d t}\left(x_{t}\right)+\int_{b}^{c} T_{d t}\left(x_{t}\right)=\int_{a}^{c} T_{d t}\left(x_{t}\right), \quad 0 \leqq a<b<c, \\
& \text { (b) } \int_{J} T_{d t}\left(x_{t}\right) \perp \int_{K} T_{d t}\left(y_{t}\right), \quad J, K \text { non overlapping, } \\
& \text { (c) }\left(\int_{J} T_{d t}\left(x_{t}\right), \int_{K} T_{d t}\left(y_{t}\right)\right)=\int_{J \cap K}\left(x_{t}, y_{t}\right) d t, \\
& \text { (d) } S_{c}\left\{\int_{a}^{b} T_{d t}\left(x_{t}\right)\right\}=\int_{a+c}^{b+c} T_{d s}\left(x_{s-c}\right) .
\end{aligned}
$$

From (5.1) and (5.3) we see that our vector-valued integral has properties akin to those possessed by stochastic integrals. ${ }^{7}$ To see the precise relationship between the two concepts, consider the function $x_{t}=c(t) \alpha$, where $\alpha \in R^{\perp}$ and $c(\cdot)$ is a complex-valued function in $L_{2}[a, b]$, and let $\xi_{t}=T_{t}(\alpha)$. Then it follows easily that the process $\left(\xi_{t}, t \geqq 0\right)$ has orthogonal increments, and

$$
\int_{a}^{b} T_{d t}\{c(t) \alpha\}=\int_{a}^{b} c(t) d \xi_{t} \quad \text { (stochastic integral). }
$$

This shows that our notion of vector-integration subsumes that of stochastic integration, but reduces to the latter when and only when $\operatorname{dim} . R^{\perp}$ $=1$.

6. The direct integral. We can now define our direct integral as a set of vector-valued integrals:

$$
\int_{a}^{b} T_{d t}\left(R^{\perp}\right)=\left\{\xi: \xi=\int_{a}^{b} T_{d t}\left(x_{t}\right), x \in L_{2}\left([a, b], R^{\perp}\right)\right\},
$$

where $0 \leqq a<b$. By $(5.1)(\mathrm{c})$, (d) this integral is a (closed) subspace of $X$. Indeed, (5.1) enables us at once to assert the following theorem:

6.2. TheOREM. The correspondence $x \rightarrow \int_{a}^{b} T_{d t}\left(x_{t}\right)$ is an isomorphism on the Hilbert space $L_{2}\left([a, b], R^{\perp}\right)$ onto the subspace $\int_{a}^{b} T_{d t}\left(R^{\perp}\right)$ of $X$, $0 \leqq a<b$.

From (5.3) we see, moreover, that as an interval-function our

${ }^{7}$ Such integrals were introduced in probability theory by Wiener, Cramer and Doob. They also occur in Hilbert space theory when spectral integrals $\int_{a}^{b} c(\lambda) d E_{\lambda}$, where $\left(E_{\lambda}, a \leqq \lambda \leqq b\right)$ is a resolution of $I$, are applied to vectors. Cf. [2, Chapter IX, $\S 2]$, and [9, Chapter VI, \$2]. 
direct integral has the following convenient properties:
(a) $\int_{a}^{b} T_{d t}\left(R^{\perp}\right)+\int_{b}^{c} T_{d t}\left(R^{\perp}\right)=\int_{a}^{c} T_{d t}\left(R^{\perp}\right), 0 \leqq a<b<c$,
(b) $\int_{J} T_{d t}\left(R^{\perp}\right) \perp \int_{K} T_{d t}\left(R^{\perp}\right), J, \quad K$ nonoverlapping,
(c) $\int_{J} T_{d t}\left(R^{\perp}\right) \subseteq \int_{K} T_{d t}\left(R^{\perp}\right), \quad J \subseteq K$,
(d) $S_{c}\left\{\int_{a}^{b} T_{d t}\left(R^{\perp}\right)\right\}=\int_{a+c}^{b+c} T_{d t}\left(R^{\perp}\right)$.

We can also show that

$$
\int_{a}^{b} T_{d t}\left(R^{\perp}\right)=\Im\left\{T_{\sigma \tau}\left(R^{\perp}\right)\right\}_{a \leqq \sigma<\tau<b} .
$$

This relation with $b=\infty$ together with (4.9) yields the result we had set out to prove:

6.5. THEOREM (WOLD DECOMPOSITION). Let $\left(S_{t}, t \geqq 0\right)$ be a strongly continuous semi-group of isometries on $X$ into $X, i H$ be its infinitesimal generator and $V$ the Cayley transform of $H$. Then for $a \geqq 0$

$$
S_{a}(x)=\int_{a}^{\infty} T_{d t}\left(R^{\perp}\right)+x_{\infty}, \quad \int_{0}^{\infty} T_{d t}\left(R^{\perp}\right) \perp x_{\infty},
$$

where $R=V(x)$ and $x_{\infty}=\bigcap_{t \geq 0} S_{t}(x)$.

From this decomposition we can readily obtain Cooper's theorem that our semi-group can be embedded in a unitary group acting on a larger Hilbert space [1, p. 841].

Our direct integral does not bear any obvious relation to the direct integral $\int_{a}^{b} \mathcal{K}_{t} d \mu(t)$ due to von Neumann and others, cf. [7], in which $\mathscr{K}_{t}$ is a Hilbert space and $\mu$ a complex-valued measure. Our integral could be written in the form $\int_{a}^{b} d \mathscr{T}_{t}$, on letting $\mathfrak{T}_{t}=T_{0 t}\left(R^{\perp}\right)$, cf. (4.4). But the significant factor in its definition is the family of operators $T_{0 t}$ and not the family of subspaces $\Re_{t}$, cf. Definitions 5.2(a), (6.1). It would seem that this integral is the tool needed for the study of the isometric representations of continuous semi-groups, just as the von Neumann integral is the tool required to deal with the unitary representations of continuous groups.

\section{REFERENCES}

1. J. L. B. Cooper, One parameter semi-groups of isometric operators in Hilbert space, Ann. of Math. 48 (1947), 827-842. 
2. J. L. Doob, Stochastic processes, Wiley, New York, 1953.

3. N. Dunford and J. Schwartz, Linear operators. I, Interscience, New York, 1958.

4. P. R. Halmos, Shifts of Hilbert spaces, Crelle's Journal 208 (1961), 102-112.

5. E. Hille and R. S. Phillips, Functional analysis and semi-groups, Amer. Math. Soc. Colloq. Publ. Vol. 31, Amer. Math. Soc., Providence, R. I., 1957.

6. P. Masani, Shift invariant spaces and prediction theory, Acta Math. 107 (1962), 275-290.

7. M. A. Naimark and S. V. Fomin, Continuous direct sums of Hilbert spaces and some of their applications, Amer. Math. Soc. Transl. (2) 5 (1957), 35-65.

8. G. Sansome, Orthogonal polynomials, Amer. Math. Soc., New York, 1959.

9. M. H. Stone, Linear transformations in Hilbert space, Amer. Math. Soc., New York, 1932.

10. N. Wiener and P. Masani, The prediction theory of multivariate stochastic processes. I, Acta Math. 98 (1957), 111-150.

\section{INDIANA UNIVERSITY}

